Pacific Journal of Mathematics

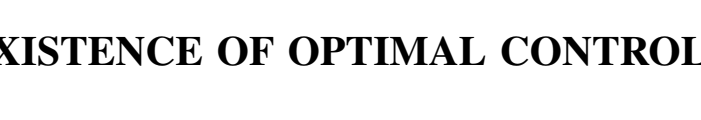




\section{EXISTENCE OF OPTIMAL CONTROLS}

\section{A. W. J. Stoddart}

Let $f=\left(f_{1}, f_{2}, \cdots, f_{n}\right)$ be a mapping to $E_{n}$ from a set $D$ in $E_{1} \times E_{n} \times E_{m}$; and $f_{0}$ a real function on $D$. Consider a "control" function $u$ from an interval $I=\left[t_{0}, t_{1}\right]$ in $E_{1}$ to $E_{m}$; and a "response" function $x$ from $I$ to $E_{n}$ such that $(t, x(t), u(t)) \in D$ for almost every $t \in I, f_{0}(t, x(t), u(t))$ has an integral (finite or $+\infty$ ) on $I, f(t, x(t), u(t)$ ) is integrable on $I$, and

$$
x(t)=x\left(t_{0}\right)+\int_{t_{0}}^{t} f(s, x(s), u(s)) d s
$$

for all $t \in I$. In a class $\Gamma$ of such control-response pairs $(u, x)$, a pair $\left(u^{*}, x^{*}\right)$ is called optimal (with respect to $f_{0}$ ) if the "cost" functional

$$
C(u, x)=(I) \int f_{0}(t, x, u) d t
$$

has a minimum at $\left(u^{*}, x^{*}\right)$. Here we consider conditions sufficient for existence of such optimal pairs.

The problem of existence of optimal controls for various functions $f, f_{0}$ and classes $\Gamma$ has been treated in [6], [11], [7], [5], [8], [9], [13], [10], [1], [2], and [3]. Gamkrelidze [6] assumed $f_{0}$ constant, $f$ linear in $(x, u)$, and $u$ restricted to a cube $U$ in $E_{m}$. Pontryagin [11] extended Gamkrelidze's work to the situation where $U$ is any compact convex polyhedron. Lee and Markus [8] considered $f$ and $f_{0}$ linear in $u$, and $U$ any compact convex set. Simple integral restraints on $u$ were treated by Krasovskii [7] and Neustadt [9].

The conditions on $U$ and $f$ for $f_{0}$ constant were relaxed remarkably by Filippov [5], who considered a variable compact restraint set $U(t, x)$ such that the set $f(t, x, U(t, x))$ is convex for each $(t, x)$. Roxin [13], in effect, considered $U$ a fixed compact set with $\left(f, f_{0}\right)(t, x, U)$ convex. By taking $f$ and $f_{0}$ linear in $x$ and $U$ compact, Neustadt [10] avoided all convexity assumptions. Cesari [1] assumes $U(t, x)$ compact, $f(t, x, U(t, x))$ convex, and $f_{0}$ sufficiently convex in $u$ compared with the curvature of $f$ in $u$. In [3], Cesari extends considerations to restraint sets $U(t, x)$ which can be unbounded.

In this paper, we consider variations of the conditions above for the case in which $f$ is linear in $u, f_{0}$ is convex in $u$, and the variable restraint set $U(t, x)$ is convex and closed but not necessarily bounded. In particular, integral restraints are taken into account, and used as an alternative source for the fundamental compactness condition. In a later section, we apply our results to classical existence problems of the calculus of variations. 
2. Definitions. We shall call a real function $\phi(t, x, u)$ "linearly bounded below in $u$ " if

$$
\phi(t, x, u) \geqq p(t, x)+u \cdot q(t, x)
$$

for some uniformly continuous and bounded functions $p, q$. The meaning of "linearly bounded in $u$ " will be obvious.

Consider the following sets, functions, and numbers.

(2) The sets $J_{0}=\left[T_{0}, T_{0}^{\prime}\right], J_{1}=\left[T_{1}, T_{1}^{\prime}\right]$ are compact intervals in $E_{1}$ with $T_{0} \leqq T_{1}^{\prime}$. Let $J=\left[T_{0}, T_{1}^{\prime}\right]$.

(3) The set $B$ is a closed set in $J \times E_{n}$, and $U$ is a closed convex set in $E_{m}$. Let $D=B \times U$.

(4) The real continuous functions $h_{j}(t, x, u)$ on $D$, at most countable in number, are convex and linearly bounded below in $u$. Let $U(t, x)=U \cap\left\{u: h_{j}(t, x, u) \leqq 0\right.$ for all $\left.j\right\}$.

(5) The mapping $G_{0}(t)$, from $J_{0}$ to the class of compact sets in $E_{n}$, is continuous in the Hausdorff sense. The mapping $G_{1}(t)$, from $J_{1}$ to the class of closed sets in $E_{n}$, is also continuous in the Hausdorff sense.

(6) The real continuous functions $g_{k}(t, x, u)$ on $D$ are convex and linearly bounded below in $u$; $c_{k}$ are corresponding real numbers.

(7) The continuous mapping $f(t, x, u)$ from $D$ to $E_{n}$ is linear in $u$ and with each component function $f_{i}$ linearly bounded in $u$. Note that linear bounding of each $f_{i}$ does not follow from linearity, even if the coefficients in $f$ are bounded; for example, $f=u \sin x^{2}$ on $E_{1} \times E_{1}$. However, if the coefficients in $f$ are bounded and each component of $u$ in $U$ is bounded above or below (in particular, $U$ bounded), then linearity implies linear bounding.

Define $\Gamma$ to be the class of all control-response pairs $(u, x)$ on intervals $I=\left[t_{0}, t_{1}\right]$, such that (1) holds, and

$$
\begin{gathered}
t_{0} \in J_{0}, \quad t_{1} \in J_{1} ; \\
(t, x(t)) \in B \quad \text { for every } t \in I ; \\
x\left(t_{0}\right) \in G_{0}\left(t_{0}\right), \quad x\left(t_{1}\right) \in G_{1}\left(t_{1}\right) ; \\
u(t) \in U(t, x(t)) \quad \text { for almost every } t \in I ; \\
(I) \int g_{k}(t, x, u) d t \leqq c_{k} \quad \text { for each } k .
\end{gathered}
$$

We shall assume that 
(13) $\int|u|$ is equi absolutely continuous on $\Gamma$;

that is, for any $\varepsilon>0$, there exists $\lambda(\varepsilon)>0$ such that $(M) \int|u| d t<\varepsilon$ for any $(u, x) \in \Gamma$ and measurable set $M \subseteq I$ for which the Lebesgue measure $\mu(M)<\lambda(\varepsilon)$. (Conditions sufficient for this will be discussed in $\S 6$.) Note that $(I) \int|u| d t$ is then bounded on $\Gamma$.

Note also that, without further restrictions on $f, x$ is not necessarily determined through (1) by $x\left(t_{0}\right)$ and $u$.

Our general approach will be to prove that the class $\Gamma$ is sequentially compact and closed in an appropriate convergence system. We then apply a general semicontinuity theorem of [14] to obtain the existence of a minimum for $C(u, x)$ on $\Gamma$.

3. A compactness theorem. We first prove a compactness theorem for $\Gamma$. It is essentially an abstraction of techniques of Tonelli [15] and Lee and Markus [8].

THeorem 1. Any infinite subclass of $\Gamma$ contains a sequence $\left(u^{n}, x^{n}\right)$ such that there exist a compact interval $I^{*}=\left[t_{0}^{*}, t_{1}^{*}\right], a$ continuous mapping $x^{*}$ from $I^{*}$ to $E_{n}$, and an integrable mapping $u^{*}$ from $I^{*}$ to $E_{m}$, for which

$$
\begin{aligned}
& \text { (a) } t_{0}^{n} \rightarrow t_{0}^{*}, t_{1}^{n} \rightarrow t_{1}^{*} ; \\
& \text { (b) } x^{n}\left(t_{0}^{n}\right) \rightarrow x^{*}\left(t_{0}^{*}\right), x^{n}\left(t_{1}^{n}\right) \rightarrow x^{*}\left(t_{1}^{*}\right) ; \\
& \text { (c) } \sup \left\{\left|x^{n}(t)-x^{*}(t)\right|: t \in I^{n} \cap I^{*}\right\} \rightarrow 0 \text {; and } \\
& \text { (d) }\left(I^{n} \cap E\right) \int u^{n} d t \rightarrow\left(I^{*} \cap E\right) \int u^{*} d t
\end{aligned}
$$

for every measurable set $E \subseteq E_{1}$.

Proof. The linear bounding of the component functions $f_{i}$ gives $|f| \leqq a+b|u|$ for some constants $a, b$. Then

$$
\left|x\left(t^{\prime}\right)-x(t)\right| \leqq a\left(t^{\prime}-t\right)+b \int_{t}^{t^{\prime}}|u| d s
$$

from (1); thus $x$ is equicontinuous on $\Gamma$.

All $G_{0}(t)$ and $J_{0}$ are compact; hence, by an elementary argument, $\mathrm{U} G_{0}(t)$ is compact. In addition, $x$ is equicontinuous and $J$ is bounded; hence $x$ is equibounded on $\Gamma$.

On $J$, define

$$
\begin{aligned}
& x_{+}(t)=x\left(t_{0}\right) \text { on }\left[T_{0}, t_{0}\right], \\
& x(t) \text { on }\left[t_{0}, t_{1}\right] \text {, } \\
& x\left(t_{1}\right) \text { on }\left[t_{1}, T_{1}^{\prime}\right] \text {; } \\
& u_{+}(t)=0 \quad \text { on }\left[T_{0}, t_{0}\right) \text { and }\left(t_{1}, T_{1}^{\prime}\right] \text {, } \\
& u(t) \text { on }\left[t_{0}, t_{1}\right] \text {. }
\end{aligned}
$$


Let $\Gamma_{+}$be the corresponding class of pairs $\left(u_{+}, x_{+}\right)$. On $\Gamma_{+}, x_{+}$is equicontinuous and equibounded, and $\int\left|u_{+}\right|$is equi absolutely continuous and $(J) \int\left|u_{+}\right| d t$ is bounded. Consequently, from any infinite subclass of $\Gamma_{+}$, we can extract in succession sequences $t_{0}^{n} \rightarrow t_{0}^{*}, t_{1}^{n} \rightarrow t_{1}^{*}$, $x_{+}^{n} \rightarrow x_{+}^{*}$ uniformly on $J$, and $u_{+}^{n} \rightarrow u_{+}^{*}$ weakly in $L_{1}(J)$ [4, p. 294] for some $t_{0}^{*}, t_{1}^{*}$, continuous $x_{+}^{*}$, and integrable $u_{+}^{*}$.

Define $x^{*}=x_{+}^{*}\left|\left[t_{0}^{*}, t_{1}^{*}\right], u^{*}=u_{+}^{*}\right|\left[t_{0}^{*}, t_{1}^{*}\right]$. Then $x^{*}$ is continuous and $u^{*}$ is integrable. Since $x_{+}^{n}\left(t_{0}^{*}\right) \rightarrow x_{+}^{*}\left(t_{0}^{*}\right), x_{+}^{n}$ is equicontinuous, and $t_{0}^{n} \rightarrow t_{0}^{*}$, we have $x^{n}\left(t_{0}^{n}\right) \rightarrow x^{*}\left(t_{0}^{*}\right)$. Similarly, $x^{n}\left(t_{1}^{n}\right) \rightarrow x^{*}\left(t_{1}^{*}\right)$.

For any $\varepsilon>0$, there exists $\lambda(\varepsilon)>0$ such that $(E) \int\left|u_{+}\right| d t<\varepsilon$ for any set $E \leqq J$ with Lebesgue measure $\mu(E)<\lambda(\varepsilon)$. Now

$$
(J) \int \phi u_{+}^{n} d t \rightarrow(J) \int \phi u_{+}^{*} d t
$$

for every $\phi \in L_{\infty}(J)$. For any measurable set $E \subseteq E_{1}$, take $\phi$ as the characteristic function of $I^{*} \cap E$. Then

$$
\left|\left(I^{*} \cap E\right) \int u_{+}^{n} d t-\left(I^{*} \cap E\right) \int u_{+}^{*} d t\right|<\varepsilon
$$

for $n$ greater than some $N(\varepsilon, E)$. Now

$$
\begin{aligned}
& \mu\left(I^{*} \cap E-I^{n} \cap E\right)+\mu\left(I^{n} \cap E-I^{*} \cap E\right) \\
& \quad \leqq\left|t_{0}^{n}-t_{0}^{*}\right|+\left|t_{1}^{n}-t_{1}^{*}\right|,
\end{aligned}
$$

which is less than $\lambda(\varepsilon)$ for $n$ greater than some $N(\varepsilon)$. Hence, for $n>N(\varepsilon)$ and $N(\varepsilon, E)$,

$$
\left|\left(I^{n} \cap E\right) \int u^{n} d t-\left(I^{*} \cap E\right) \int u^{*} d t\right|<2 \varepsilon .
$$

4: Continuity and semicontinuity. The following continuity theorem is required for the semicontinuity theorem.

Theorem 2. Let $\left(u^{n}, x^{n}\right)$ be a sequence in $\Gamma$ converging to $\left(u^{*}, x^{*}\right)$ in the sense (14). Let the functions $p: B \rightarrow E_{1}$ and $q: B \rightarrow E_{m}$ be uniformly continuous and bounded. Then, for every measurable set $E \subseteq E_{1}$,

$$
\begin{aligned}
\left(I^{n} \cap E\right) \int\left[p\left(t, x^{n}\right)+u^{n} \cdot q\left(t, x^{n}\right)\right] d t & \\
& \rightarrow\left(I^{*} \cap E\right) \int\left[p\left(t, x^{*}\right)+u^{*} \cdot q\left(t, x^{*}\right)\right] d t .
\end{aligned}
$$

Proof. Note that, since $B$ is closed, conditions (14) (a), (b), (c) ensure that $\left(t, x^{*}(t)\right) \in B$ for every $t \in I^{*}$. 
We express our conditions in explicit form. For any $\varepsilon>0$, there exists $N(\varepsilon)$ such that, for $n>N(\varepsilon)$,

$$
\begin{aligned}
& \left|t_{0}^{n}-t_{0}^{*}\right|<\varepsilon, \\
& \left|t_{1}^{n}-t_{1}^{*}\right|<\varepsilon, \\
& \left|x^{n}\left(t_{0}^{n}\right)-x^{*}\left(t_{0}^{*}\right)\right|<\varepsilon, \\
& \left|x^{n}\left(t_{1}^{n}\right)-x^{*}\left(t_{1}^{*}\right)\right|<\varepsilon,
\end{aligned}
$$

and

$$
\left|x^{n}(t)-x^{*}(t)\right|<\varepsilon
$$
if $t \in I^{n} \cap I^{*}$;

and, for any measurable set $E \subseteq E_{1}$, there exists $N(\varepsilon, E)$ such that, for $n>N(\varepsilon, E)$,

$$
\left|\left(I^{n} \cap E\right) \int u^{n} d t-\left(I^{*} \cap E\right) \int u^{*} d t\right|<\varepsilon .
$$

Also, there exists $\lambda(\varepsilon)>0$ such that $(M) \int\left|u^{n}\right| d t<\varepsilon$ for all $n$ and for every measurable set $M \cong I^{n}$ with $\mu(M)<\lambda(\varepsilon)$; and there exists $\alpha$ such that $\left(I^{n}\right) \int\left|u^{n}\right| d t<\alpha$ for all $n$. In addition, there exists $\delta(\varepsilon)>0$ such that $\left|p(t, x)-p\left(t^{\prime}, x^{\prime}\right)\right|$ and $\left|q(t, x)-q\left(t^{\prime}, x^{\prime}\right)\right|<\varepsilon$ for $\left|t-t^{\prime}\right|$ and $\left|x-x^{\prime}\right|<\delta(\varepsilon)$; and there exists $\beta$ such that $|p(t, x)|$ and $|q(t, x)|<\beta$ for all $(t, x) \in B$.

Now

$$
\begin{aligned}
& \left|\left(I^{n} \cap E\right) \int p\left(t, x^{n}\right) d t-\left(I^{*} \cap E\right) \int p\left(t, x^{*}\right) d t\right| \\
& \quad \leqq \beta\left(\left|t_{1}^{n}-t_{1}^{*}\right|+\left|t_{0}^{n}-t_{0}^{*}\right|\right) \\
& \quad+\left(I^{n} \cap E \cap I^{*}\right) \int\left|p\left(t, x^{n}\right)-p\left(t, x^{*}\right)\right| d t \\
& \quad<\beta \varepsilon+\left(T_{1}^{\prime}-T_{0}\right) \varepsilon \quad \text { if } n>N\left(\frac{1}{2} \varepsilon\right) \text { and } N(\delta(\varepsilon)) .
\end{aligned}
$$

Also,

$$
\begin{aligned}
& \left|\left(I^{n} \cap E\right) \int u^{n} \cdot q\left(t, x^{n}\right) d t-\left(I^{n} \cap E \cap I^{*}\right) \int u^{n} \cdot q\left(t, x^{*}\right) d t\right| \\
& \quad \leqq \beta\left(I^{n}-I^{*}\right) \int\left|u^{n}\right| d t \\
& \quad+\left|\left(I^{n} \cap E \cap I^{*}\right) \int u^{n} \cdot\left[q\left(t, x^{n}\right)-q\left(t, x^{*}\right)\right] d t\right| \\
& \quad<\beta \varepsilon+\alpha \varepsilon \quad \text { if } n>N\left(\frac{1}{2} \lambda(\varepsilon)\right) \text { and } N(\delta(\varepsilon)) .
\end{aligned}
$$

By uniform continuity of $x^{*}$ on $I^{*}$, there exists $\gamma(\varepsilon)>0$ such that $\left|x^{*}(t)-x^{*}\left(t^{\prime}\right)\right|<\varepsilon$ for $\left|t-t^{\prime}\right|<\gamma(\varepsilon)$. Divide $I^{*}$ into $\sigma$ intervals $I_{s}$ 
with lengths less than $\delta(\varepsilon)$ and $\gamma(\delta(\varepsilon))$, and take $q_{s}=q\left(t, x^{*}(t)\right)$ for some $t \in I_{s}$. Then

$$
\begin{aligned}
\left|\left(I^{n} \cap E \cap I^{*}\right) \int u^{n} \cdot q\left(t, x^{*}\right) d t-\left(I^{*} \cap E\right) \int u^{*} \cdot q\left(t, x^{*}\right) d t\right| \\
=\left|\sum\left(I^{n} \cap E \cap I_{s}\right) \int u^{n} \cdot q\left(t, x^{*}\right) d t-\sum\left(I_{s} \cap E\right) \int u^{*} \cdot q\left(t, x^{*}\right) d t\right| \\
<\left|\sum q_{s} \cdot\left[\left(I^{n} \cap E \cap I_{s}\right) \int u^{n} d t-\left(I_{s} \cap E\right) \int u^{*} d t\right]\right| \\
\quad+\varepsilon\left(I^{*}\right) \int\left|u^{*}\right| d t+\alpha \varepsilon \\
<\varepsilon+\varepsilon\left(I^{*}\right) \int\left|u^{*}\right| d t+\alpha \varepsilon \quad \text { if } n>\max N\left(\varepsilon / \sigma\left|q_{s}\right|, I_{s} \cap E\right) .
\end{aligned}
$$

We shall use repeatedly the following semicontinuity theorem.

THEOREM 3. Let $\phi(t, x, u)$ be a real continuous function on $D$, convex and linearly bounded below in $u$. Consider a sequence $\left(u^{n}, x^{n}\right) \in \Gamma$ converging to $\left(u^{*}, x^{*}\right)$ in the sense (14). (We shall prove in $\S 5$ that $u^{*}(t) \in U$ for almost every $t \in I^{*}$; and $\left(t, x^{*}(t)\right) \in B$ for every $t \in I^{*}$.) Then, for every measurable set $E \leqq E_{1}$,

$$
\left(I^{*} \cap E\right) \int \phi\left(t, x^{*}, u^{*}\right) d t \leqq \liminf \left(I^{n} \cap E\right) \int \phi\left(t, x^{n}, u^{n}\right) d t .
$$

Theorem 3 follows easily from Theorem 4 of [14]. Our convergence (14) satisfies condition (10) of [14]. The discussion of $\S 6$ of [14] applies here, since the lower bound integral is continuous.

\section{A closure theorem.}

Theorem 4. Let $\left(u^{n}, x^{n}\right)$ be a sequence in $\Gamma$ converging to $\left(u^{*}, x^{*}\right)$ in the sense (14). Then $\left(u^{*}, x^{*}\right) \in \Gamma$.

Proof. By (14a), $t_{0}^{*} \in J_{0}$ and $t_{1}^{*} \in J_{1}$.

For $t_{0}^{*}<t<t_{1}^{*}, t \in I^{n}$ for all sufficiently large $n$, so $x^{n}(t) \rightarrow x^{*}(t)$ by (14c). Thus $\left(t, x^{*}(t)\right) \in B$. In addition, (14a) and (14b) give $\left(t_{0}^{*}, x^{*}\left(t_{0}^{*}\right)\right)$ and $\left(t_{1}^{*}, x^{*}\left(t_{1}^{*}\right)\right) \in B$.

If $x^{*}\left(t_{0}^{*}\right)$ were not in $G_{0}\left(t_{0}^{*}\right)$, then it would not be in the closure $N^{c}$ of some neighbourhood $N$ of $G_{0}\left(t_{0}^{*}\right)$. But $G_{0}(t) \subseteq N$ for $t$ sufficiently near $t_{0}^{*}$, so $x^{n}\left(t_{0}^{n}\right) \in N$ for all sufficiently large $n$, from which $x^{*}\left(t_{0}^{*}\right) \in N^{c}$ ! Similarly, $x^{*}\left(t_{1}^{*}\right) \in G_{1}\left(t_{1}^{*}\right)$.

The closed convex set $U$ in $E_{m}$ is the intersection of a countable number of half spaces $\{u: \beta+u \cdot b \leqq 0\}$. Let

$$
E=\left\{t: t \in I^{*}, \beta+u^{*}(t) \cdot b>0\right\} .
$$


Then

$$
0 \geqq\left(I^{n} \cap E\right) \int\left(\beta+u^{n} \cdot b\right) d t \rightarrow(E) \int\left(\beta+u^{*} \cdot b\right) d t \geqq 0 .
$$

Thus $\mu(E)=0$, and so $u^{*}(t) \in U$ for almost every $t \in I^{*}$.

Let $E_{j}=\left\{t: t \in I^{*}, h_{j}\left(t, x^{*}(t), u^{*}(t)\right)>0\right\}$. Now

$$
\left(I^{n} \cap E_{j}\right) \int h_{j}\left(t, x^{n}, u^{n}\right) d t \leqq 0,
$$

so

$$
\left(E_{j}\right) \int h_{j}\left(t, x^{*}, u^{*}\right) d t \leqq 0
$$

by Theorem 3. Consequently, $\mu\left(E_{j}\right)=0$. Thus $\left(u^{*}, x^{*}\right)$ satisfies (11).

By Theorem 3 with $E=E_{1}$, the integral $(I) \int g_{k}(t, x, u) d t$ is lower semicontinuous in the convergence (14). Consequently

$$
\left(I^{*}\right) \int g_{k}\left(t, x^{*}, u^{*}\right) d t \leqq c_{k},
$$

that is, condition (12) is satisfied.

Consider $t$ such that $t_{0}^{*}<t<t_{1}^{*}$. Theorem 3 with $E=\{s: s \leqq t\}$ shows that the integral

$$
\int_{t_{0}}^{t} f(s, x(s), u(s)) d s
$$

is continuous in the convergence (14). Also, $x^{n}(t) \rightarrow x^{*}(t)$ and $x^{n}\left(t_{0}^{n}\right) \rightarrow$ $x^{*}\left(t_{0}^{*}\right)$. Thus condition (1) on $\left(u^{n}, x^{n}\right)$ carries over to $\left(u^{*}, x^{*}\right)$. For $t=t_{1}^{*}$, a similar argument applies, but with $E=E_{1}$.

Thus $\left(u^{*}, x^{*}\right)$ satisfies conditions (1) and (8) through (12).

\section{The existence theorem.}

THEOREM 5. Let the real continuous function $f_{0}(t, x, u)$ on $D$ be convex and linearly bounded below in $u$. Assume, as previously, that $\Gamma$ satisfies condition (13). Then, if $\Gamma$ is not empty, $C(u, x)$ has a minimum on $\Gamma$.

Proof. Theorems 1 and 4 show that $\Gamma$ is sequentially compact in itself with respect to the convergence (14).

Since $f_{0}$ is linearly bounded below in $u$ and $u$ is integrable, $f_{0}(t, x, u)$ has an integral, finite or $+\infty$. Theorem 3 , with $E=E_{1}$, shows that $(I) \int f_{0}(t, x, u) d t$ is lower semicontinuous with respect to the convergence (14). 
A lower semicontinuous funotional on a sequentially compact space has a minimum. Hence the result.

7. Equi absolute continuity of $\int|u|$. Condition (13) plays the key part in our compactness theorem. We now study conditions sufficient for equi absolute continuity of $\int|u|$ on $\Gamma$.

For example, if the set $U$ and the functions $h_{j}$ are such that

$$
U(t, x)=U \cap\left\{u: h_{j}(t, x, u) \leqq 0 \text { for all } j\right\}
$$

is bounded uniformly on $B$, then condition (13) is obviously satisfied. This is the standard situation in problems of optimal control.

The following more general integral condition is quite standard in the calculus of variations.

THEOREM 6. Let $\psi(u)$ be a real function on $E_{m}$, bounded below and such that $\psi(u) /|u| \rightarrow \infty$ as $|u| \rightarrow \infty$. If $(I) \int \psi(u(t)) d t$ is bounded on $\Gamma$, then $\int|u|$ is equi absolutely continuous.

Proof. Suppose that $(I) \int \psi(u) d t \leqq c$ on $\Gamma$; $\psi(u) \geqq b$; and, for any $\varepsilon>0,(\psi(u)-b) /|u|>1 / \varepsilon$ for $|u|>m(\varepsilon)$. For any $(u, x) \in \Gamma$ and measurable set $M \subseteq I$, define

$$
M^{+}=M \cap\{t: t \in I,|u(t)|>m(\varepsilon)\}, \quad M^{-}=M-M^{+} .
$$

Then

$$
\begin{aligned}
(M) \int|u(t)| d t & =\left(M^{+}\right) \int|u| d t+\left(M^{-}\right) \int|u| d t \\
& \leqq \varepsilon\left(M^{+}\right) \int(\psi(u)+|b|) d t+m(\varepsilon) \mu\left(M^{-}\right) \\
& \leqq \varepsilon(I) \int(\psi(u)+|b|) d t+m(\varepsilon) \mu(M) \\
& <\varepsilon\left(c+|b|\left(T_{1}^{\prime}-T_{0}\right)+1\right)
\end{aligned}
$$

if $\mu(M)<\varepsilon / m(\varepsilon)$. Thus $\int|u|$ is equi absolutely continuous.

For example, a "growth condition" $g_{k}(t, x, u) \geqq \psi(u)$ on some $g_{k}$ would be sufficient for the bounding of $(I) \int \psi(u) d t$ on $\Gamma$. Alternatively, the bounding of $(I) \int \psi(u) d t$, sufficiently for our purpose, would follow from a similar growth condition on $f_{0}$.

THEOREM 7. Suppose that $f_{0}(t, x, u) \geqq \psi(u)$, where $\psi$ has the properties stated in Theorem 6. Then our existence theorem, Theorem 5, holds without the direct assumption of condition (13). 
Proof. If $C(u, x)=\infty$ for all $(u, x) \in \Gamma$, then the result is trivial. Otherwise, there exists $\left(u_{1}, x_{1}\right) \in \Gamma$ with $C\left(u_{1}, x_{1}\right)<\infty$. In considering a minimum for $C(u, x)$ on $\Gamma$, we can restrict consideration to the class

$$
\Gamma_{1}=\Gamma \cap\left\{(u, x): C(u, x) \leqq C\left(u_{1}, x_{1}\right)\right\} .
$$

Then $(I) \int \psi(u) d t$ is bounded on $\Gamma_{1}$. Theorems 5 and 6 show that $C(u, x)$ has a minimum on $\Gamma_{1}$, which is obviously also a minimum on $\Gamma$.

8. Extension to unbounded intervals $J_{0}, J_{1}$, If $J_{1}$ has semiinfinite form, then our existence theorem still holds, provided $f_{0}$ is positively bounded below.

THEOREM 8. Assume that $f_{0}(t, x, u) \geqq m$ for some positive constant $m$. Then Theorm 5 holds also for $J_{1}$ of the form $\left[T_{1}, \infty\right)$.

Proof. If $C(u, x)=\infty$ for all $(u, x) \in \Gamma$, then the existence of a minimum for $C(u, x)$ is trivial. Otherwise, $C_{1}=C\left(u_{1}, x_{1}\right)<\infty$ for some $\left(u_{1}, x_{1}\right) \in \Gamma$. We can restrict consideration to the class $\Gamma_{1}$ of those $(u, x) \in \Gamma$ for which $C(u, x) \leqq C_{1}$.

For $(u, x) \in \Gamma_{1}, C_{1} \geqq C(u, x) \geqq m\left(t_{1}-t_{0}\right)$, so

$$
t_{1} \leqq t_{0}+C_{1} / m \leqq T_{0}^{\prime}+C_{1} / m \text {. }
$$

Thus the condition $t_{1} \in\left[T_{1}, T_{0}^{\prime}+C_{1} / m\right]$ does not further restrict $\Gamma_{1}$. Then Theorem 5 shows that $C(u, x)$ has a minimum on $\Gamma_{1}$, which is also a minimum on $\Gamma$.

Obviously, similar considerations apply when $J_{0}=\left(-\infty, T_{0}^{\prime}\right]$; and, indeed, when $J_{0}$ and $J_{1}$ both have these semi-infinite forms.

9. Classical problems. If $U=E_{m}$ and the class of functions $h_{j}$ is empty, then $U(t, x)=E_{m}$ for all $(t, x)$, that is, there are no explicit restrictions (11). In this case, the fundamental condition (13) on $u$ could come from a growth condition on $f_{0}$ or some $g_{k}$, as discussed in $\S 7$.

If we take $f(t, x, u)=u$, so that $u=x^{\prime}$ almost everywhere, then we have a minimum problem for $(I) \int f_{0}\left(t, x, x^{\prime}\right) d t$. The Tonelli theorem [16], on the existence of a minimum for nonparametric curve integrals, is just this problem with no explicit restrictions (11) and no integral restrictions (12); the condition (13) comes from a growth condition on $f_{0}$.

More generally, consider curves $y: I \rightarrow E_{l}$ with absolutely continuous derivatives $y^{(r-1)}$ of order $r-1$. Take $x=\left(x_{(1)}, x_{(2)}, \cdots, x_{(r)}\right)$ 
with $x_{(\alpha)}(t)=y^{(\alpha-1)}(t)$, and $u(t)=y^{(r)}(t)$. Then our work gives an existence theorem for the minimum of $(I) \int f_{0}\left(t, y, y^{\prime}, \cdots, y^{(r)}\right) d t$. Here

$$
f(t, x, u)=\left(x_{(i)}, x_{(3)}, \cdots, x_{(r)}, u\right) .
$$

The linear bounding of the components of $f$ is implied essentially by the bounding of $(I) \int\left|y^{(r)}\right| d t$.

Returning to first order problems, we can also consider parametric curve integrals $(I) \int f_{0}\left(x, x^{\prime}\right) d t$ with $f_{0}$ positively homogeneous of degree one in $x^{\prime}$. In this case, a growth condition on $f_{0}$ of the form previously considered is impossible. However, if there are no explicit restrictions (11), the functions $g_{k}$ are similarly independent of $t$ and positively homogeneous of degree one in $x^{\prime}, G_{0}$ and $G_{1}$ are constant, and $B$ is of the form $E_{1} \times C$ for some closed set $C$ in $E_{n}$, then we have a system invariant under Fréchet equivalence. We can reparametrize the curves of finite length $L \neq 0$ by their relative arc lengths $s / L$ on the interval $I=[0,1]$; here $s$ is the arc length. In terms of the new parameter, $\left|x^{\prime}\right|=L$ almost everywhere. If the curves in $\Gamma$ have bounded lengths, then $\int\left|x^{\prime}\right|$ is equi absolutely continuous. This is trivial for curves with $L=0$. Thus condition (13) would be satisfied if the curves have bounded lengths.

We have really proved here part of Hilbert's theorem on compactness of a class of parametric curves. The bounding of the curve lengths $L$ could come from the form of some $g_{k}$

$$
\text { (for example, }(I) \int g_{k}\left(x, x^{\prime}\right) d t \rightarrow \infty \text { as } L \rightarrow \infty \text { ); }
$$

or, effectively, from the form of $f_{0}$

$$
\text { (for example, }(I) \int f_{0}\left(x, x^{\prime}\right) d t \rightarrow \infty \text { as } L \rightarrow \infty \text { ). }
$$

\section{REFERENCES}

1. L. Cesari, Un teorema di esistenza in problemi di controlli ottimi, Ann. Scuola Norm. Sup. Pisa 19 (1965), 35-78.

2. - An existence theorem in problems of optimal control, J. STAM Control (A) 3 (1965), 7-22.

3. - Existence theorems for optimal solutions in Pontryagin and Lagrange problems, J. SIAM Control (A) 3 (1966), 475-498.

4. N. Dunford and J. T. Schwartz, Linear Operators, Part I, Interscience Publishers, New York, 1958.

5. A. F. Filippov, On certain questions in the theory of optimal control, Vestnik Moskov. Univ. Ser. I Mat. Meh. 2 (1959), 25-32 (Russian). English translation in J. SIAM Control (A) 1 (1962), 76-84.

6. R. V. Gamkrelidze, The theory of time-optimal processes in linear systems, Izv. Akad. Nauk SSSR. Ser. Mat. 22 (1958), 449-474 (Russian). 
7. N. N. Krasovskii, On the theory of optimal control, Avtomat. i Telemeh. 18 (1957), 960-970 (Russian). English translation in Automation and Remote Control 18 (1957), 1005-1016.

8. E. B. Lee and L. Markus, Optimal control for nonlinear processes, Arch. Rational Mech. Anal. 8 (1961), 36-58.

9. L. W. Neustadt, Time optimal control systems with position and integral limits, J. Math. Anal. Appl. 3 (1961), 406-427.

10. The existence of optimal controls in the absence of convexity conditions, J. Math. Anal. Appl. 7 (1963), 110-117.

11. L. S. Pontryagin, Optimal control processes, Uspehi Mat. Nauk 14 (1959), 3-20 (Russian). English translation in Automation Express 2 (1959), 26-30.

12. L. S. Pontryagin, V. G. Boltyanskii, R. V. Gamkrelidze, and E. F. Mishchenko, The Mathematical Theory of Optimal Processes (English translation), Interscience Publishers, New York, 1962.

13. E. Roxin, The existence of optimal controls, Michigan Math. J. 9 (1962), 109-119. 14. A. W. J. Stoddart, Semicontinuity of integrals, Trans. Amer. Math. Soc. 122 (1966), 120-135.

15. L. Tonelli, Fondamenti di Calcolo delle Variazioni. 2 vols. Zanichelli, Bologna, 1921-23.

16. - Su gli integrali del calcolo delle variazioni in forma ordinaria, Ann. Scuola Norm. Sup. Pisa 3 (1934), 401-450. Opere Scelte, Cremonese, Roma (1962) 3, 192-254.

Received December 8, 1965.

THE UNIVERSITY OF OTAGo,

DUNEDin, NeW Zealand 



\section{PACIFIC JOURNAL OF MATHEMATICS}

H. SAMELSON

Stanford University

Stanford, California

J. P. JaNs

University of Washington

Seattle, Washington 98105

\section{EDITORS}

J. DugundJI

University of Southern California Los Angeles, California 90007

RICHARD ARENS

University of California

Los Angeles, California 90024

\section{ASSOCIATE EDITORS}

E. F. BECKENBACH

B. H. NEUMANN

F. WOLF

K. YosidA

\section{SUPPORTING INSTITUTIONS}

UNIVERSITY OF BRITISH COLUMBIA

CALIFORNIA INSTITUTE OF TECHNOLOGY

UNIVERSITY OF CALIFORNIA

MONTANA STATE UNIVERSITY

UNIVERSITY OF NEVADA

NEW MEXICO STATE UNIVERSITY

OREGON STATE UNIVERSITY

UNIVERSITY OF OREGON

OSAKA UNIVERSITY

UNIVERSITY OF SOUTHERN CALIFORNIA
STANFORD UNIVERSITY

UNIVERSITY OF TOKYO

UNIVERSITY OF UTAH

WASHINGTON STATE UNIVERSITY

UNIVERSITY OF WASHINGTON

AMERICAN MATHEMATICAL SOCIETY CHEVRON RESEARCH CORPORATION TRW SYSTEMS

NAVAL ORDNANCE TEST STATION 


\section{Pacific Journal of Mathematics}

\section{Vol. 20, No. $1 \quad$ September, 1967}

Leonard Daniel Baumert, Extreme copositive quadratic forms. II ........ 1

Edward Lee Bethel, A note on continuous collections of disjoint

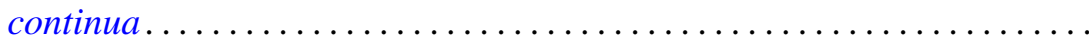

Delmar L. Boyer and Adolf G. Mader, A representation theorem for abelian groups with no elements of infinite p-height ...................

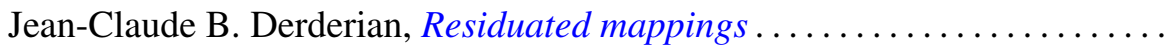

Burton I. Fein, Representations of direct products of finite groups ......... 45

John Brady Garnett, A topological characterization of Gleason parts.......

Herbert Meyer Kamowitz, On operators whose spectrum lies on a circle or

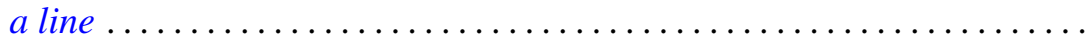

Ignacy I. Kotlarski, On characterizing the gamma and the normal distribution ........................................

Yu-Lee Lee, Topologies with the same class of homeomorphisms ..........

Moshe Mangad, Asymptotic expansions of Fourier transforms and discrete polyharmonic Green's functions ...........................

Jürg Thomas Marti, On integro-differential equations in Banach spaces ....

Walter Philipp, Some metrical theorems in number theory.............. 109

Maxwell Alexander Rosenlicht, Another proof of a theorem on rational

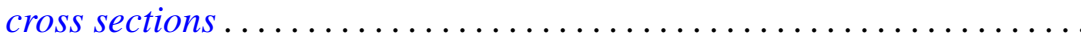

Kenneth Allen Ross and Karl Robert Stromberg, Jessen's theorem on Riemann sums for locally compact groups

Stephen Simons, A theorem on lattice ordered groups, results of Ptak, Namioka and Banach, and a front-ended proof of Lebesgue's theorem...

Morton Lincoln Slater, On the equation $\varphi(x)=\int_{x} x+1 K(\xi) f[\varphi(\xi)] d \xi \ldots$ 155 Arthur William John Stoddart, Existence of optimal controls .. 167 Burnett Roland Toskey, A system of canonical forms for rings on a direct sum of two infinite cyclic groups ....................

Jerry Eugene Vaughan, A modification of Morita's characterization of dimension 